\title{
Failure of once daily inhaled corticosteroid treatment to control chronic asthma
}

\author{
DV McGIVERN, M WARD, JT MACFARLANE, WH RODERICK SMITH \\ From the Department of Thoracic Medicine, City Hospital, Nottingham
}

\begin{abstract}
Twelve patients with chronic asthma received either high dose beclomethasone once a day or standard doses of beclomethasone three times a day in a double blind crossover trial to determine whether inhalation once a day would be sufficient. With the once a day regimen peak expiratory flow rates fell significantly, symptoms of nocturnal asthma increased, and two patients withdrew from the study because of worsening asthma. Whether control can be achieved with steroids inhaled once a day by simply increasing the total daily dose remains to be seen.
\end{abstract}

Inhaled corticosteroids used in treating chronic asthma are usually given three or four times daily, ${ }^{\prime}$ but recently it has been shown that twice daily regimens may also be effective..$^{23}$ To see whether inhalation once a day would be sufficient we compared standard dose inhaled beclomethasone administered by metered dose inhaler (Becotide, $50 \mu \mathrm{g}$ per puff), given three or four times daily, with an equivalent daily dose given once each morning from a high dose beclomethasone inhaler (Becloforte, $250 \mu \mathrm{g}$ per puff).

\section{Methods}

\section{PATIENTS}

Twelve patients, aged $22-71$, with chronic asthma gave their informed consent to participate in this study. All were receiving both inhaled beclomethasone and $\beta$ stimulants regularly from pressurised aerosols. Patients taking oral corticosteroids were excluded. The technique of inhalation was checked and standardised before the patient was included in the study and was rechecked at each clinic attendance.

\section{METHODS}

A double blind crossover study was performed. Patients were observed during a run in period of two weeks, during which they took their usual drugs. If their asthma was stable they were then studied dur-

\footnotetext{
Address for reprint requests: Dr DV McGivern, Department of Thoracic Medicine, City Hospital, Nottingham NG5 1PB.
}

Accepted 18 June 1984 ing two consecutive periods of four weeks each while taking one or other of the following regimens: high dose beclomethasone once each morning plus placebo inhaler in divided daily doses; or standard beclomethasone in divided daily doses plus placebo inhaler once each morning. All active and placebo inhalers were the same plain colour, labelled with dosage instructions. The order of receiving the two regimens was randomised. Standard beclomethasone (or placebo) was given in three or four divided daily doses as usual and the total daily dose was not changed. The dosage of once daily, high dose beclomethasone (or placebo) was calculated as follows: 4-6 puffs standard beclomethasone/day $(200-300 \mu \mathrm{g})=1$ puff high dose $(250 \mu \mathrm{g}) ; 7-10$ puffs standard beclomethasone/day $(350-500 \mu \mathrm{g})=$ 2 puffs high dose $(500 \mu \mathrm{g}) ; 11$ and 12 puffs standard beclomethasone/day ( 550 and $600 \mu \mathrm{g})=3$ puffs high dose $(750 \mu \mathrm{g})$. Inhaled or oral bronchodilators were taken as usual.

The patients kept a diary and recorded scores for daytime and nocturnal symptoms and peak expiratory flow rate (PEFR). To check compliance with treatment doses of all drugs used were also recorded. Symptoms of daytime wheeze, shortness of breath, and nocturnal asthma were scored by the patients as none (0), mild (1), moderate (2), or severe (3). Patients recorded peak expiratory flow rate with a Wright mini peak flow meter before taking bronchodilators on rising and before going to bed. The best of three attempts was recorded. At each visit to the clinic patients were asked about sore throat and the oropharynx was inspected. Swabs were taken from any areas that appeared to be suggestive of oral candidiasis and cultures made. 


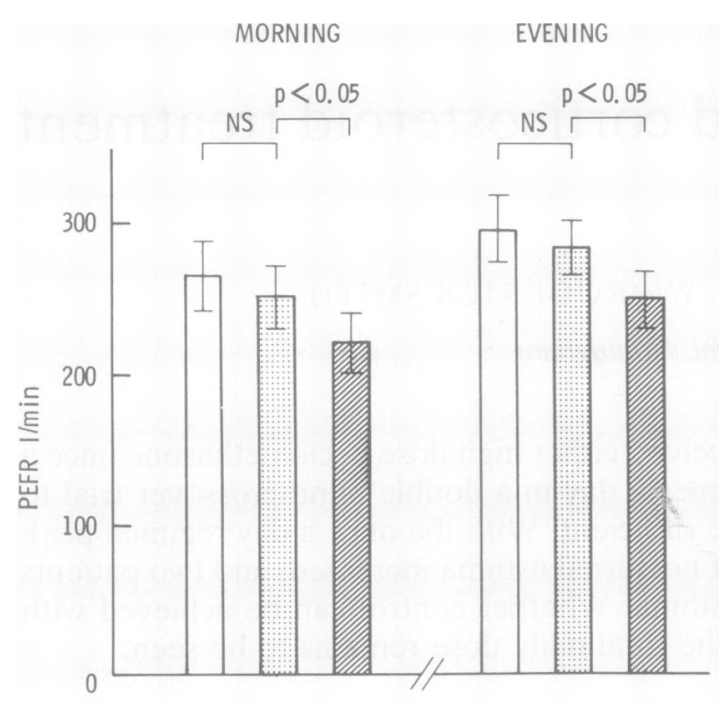

NOCTURNAL ASTHMA DAYTIME WHEEZE SHORTNESS OF BREATH

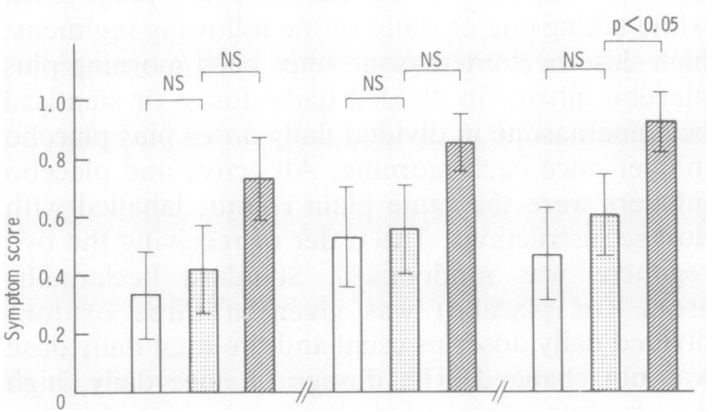

Mean (SE) peak expiratory flow rate (PEFR) (top) and symptom scores (bottom) in 10 patients with chronic asthma. Results shown are for the run in period ( $\square$ ) and for four weeks taking either standard beclomethasone (国) or once daily high dose beclomethasone (1).

STATISTICAL ANALYSIS

During each period mean scores for symptoms and peak expiratory flow rate were calculated, and these were compared using a paired $t$ test. To allow for a possible "carry over" effect after changing regimens data for the first 10 days of each four week period were not analysed.

\section{Results}

Two patients withdrew from the study because of increasing asthma seven and 10 days after starting the once daily regimen. In the remaining 10 patients there was no difference in scores for symptoms and peak expiratory flow rate between the run in period and the four weeks of standard regimen but significant deterioration occurred during once daily treatment (figure). Two patients developed symptomatic oral candidiasis (confirmed by culture), while one was receiving the standard and the other the high dose treatment.

\section{Discussion}

Two studies have shown that inhaled corticosteroids given twice daily produce satisfactory control of $x$ asthma. ${ }^{23}$ In our study, however, beclomethasone $\omega$ inhaled once a day was less effective than an equi- $\vec{N}$ valent amount given in three or four divided doses. Peak expiratory flow rate fell significantly, symp- $\omega$ toms of nocturnal asthma increased, and two응 patients withdrew because of worsening asthmawhen patients were changed to a once daily regimen. Although shortness of breath and daytime wheeze also increased, these just failed to achieve significance, possibly because patients found these symptoms more difficult to measure.

We elected to given once daily treatment from $a_{\infty}$ high dose beclomethasone inhaler rather than by. multiple inhalations from a standard dose inhaler because this would be a more convenient and practical method for the patient. Although this meant thato an identical dose of beclomethasone was not giveno in the two periods of treatment, the dose conversiono we used ensured that in all but one of the patients the once daily dose was somewhat higher. Deterio-3 ration while receiving once daily treatment was thus not due to a reduction in the effective daily dose.

In contrast with these findings with inhaled cor ticosteroids, patients taking oral corticosteroids areo usually satisfactorily controlled with once daily of alternate day dosage. Whether control can be achieved with once daily inhaled steroids by simply? increasing the total daily dose remains to be seen.

We are grateful to Glaxo Pharmaceutical for provid-o ing active and placebo inhalers.

\section{References}

1 Gaddie J, Reid IW, Skinner C, Petrie GR, Sinclaif DJM, Palmer KNV. Aerosol beclomethasone dipfN ropionate: a dose-response study in chronic asthma Lancet 1973;ii:280-1.

2 Munch EP, Taudorf E, Wecke B. Dose frequency ifP

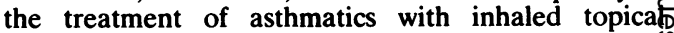
steroids. Eur J Respir Dis 1982;63:143.

3 Willey RF, Godden DJ, Carmichael J, Preston $P_{7}$ Frame M, Crompton GK. Comparison of twice dail $\bar{b}$ administration of a new corticosteroid budesonide witle beclomethasone dipropionate four times daily in the treatment of chronic asthma. $\mathrm{Br} J$ Dis Ches 1982;76:61-8. 